\title{
Las elecciones legislativas de Colombia de 2018: un análisis de las publicaciones de los principales cibermedios colombianos en Facebook y Twitter
}

\begin{abstract}
Resumen
A través de las redes sociales, los medios en línea tienen la oportunidad no solo de distribuir los contenidos que originan, sino también de interactuar con sus audiencias y fomentar, con ello, un mayor diálogo social. Esto es especialmente relevante en las rutinas electorales, en que los candidatos y los partidos se encargan de presentar sus programas ante sus electores. Con este artículo, y a través de un análisis de contenido, se han evaluado 1272 mensajes difundidos a través de las cuentas de Twitter y Facebook de ocho cibermedios colombianos de cobertura nacional durante las elecciones legislativas de ese país de 2018. Entre los resultados principales, encontramos un protagonismo político dado, sobre todo, a las principales organizaciones políticas, además de unos tratamientos, en general, más favorables a estas mismas organizaciones.
\end{abstract}

\author{
Daniel Barredo Ibáñez \\ PhD. en Periodismo. \\ Profesor de carrera de la Universidad \\ del Rosario, Bogotá - Colombia. \\ Investigador invitado en la Fudan \\ University, Shanghái - China. \\ Correo electrónico: \\ daniel.barredo@urosario.edu.co \\ ๑ orcid.org/0000-0002-2259-0756 \\ Google Scholar
}

\author{
Sandra Lucía Ruiz Moreno \\ Mg. en Dirección de la empresa \\ audiovisual \\ Decana de la Facultad de \\ Comunicación de la Universidad \\ Santo Tomás \\ Bogotá - Colombia \\ Correo electrónico: \\ dec.comunicacionsocial@ \\ usantotomas.edu.co \\ ๑ orcid.org/0000-0001-5578-3194 \\ Google Scholar
}

Recibido: mayo 17 de 2019

Aprobado: octubre 14 de 2020

Palabras clave:

Twitter, Facebook, participación ciudadana, Colombia. 


\title{
The 2018 Colombian legislative elections: an analysis of the publications of the main Colombian cybermedia on Facebook and Twitter
}

\begin{abstract}
Through social networks, online media have the opportunity not only to distribute the content they originate, but also to interact with their audiences and thereby foster greater social dialogue. This is especially relevant in electoral routines in which candidates and parties are in charge of presenting their programs to their electors. With this article, and through a content analysis, a total of 1,272 messages disseminated through the Twitter and Facebook accounts of eight Colombian cybermedia with national coverage during the 2018 Colombian legislative elections have been evaluated. Among the main results, we find a political prominence given, above all, to the main political organizations, in addition to a more favorable treatment in general to these organizations.
\end{abstract}




\section{Introducción}

Las redes sociales están contribuyendo a la configuración de una mayor diversidad e inclusión (Ríos et al., 2017), al favorecer la aparición de nuevas formas de discusión (Skogerbø \& Krumsvik, 2015) y al conformar espacios participados por las personas, las organizaciones e instituciones, y los medios de comunicación (Barredo Ibáñez, 2021). Con todo, las redes sociales tienen una naturaleza controversial: si, por un lado, han incentivado nuevos repertorios de activismo político, lo cierto es que Harlow \& Guo (2014) advierten que también han contribuido a su erosión, con la aparición de los Ilamados "clicktivistas" (p. 475), es decir, activistas cuya movilización no necesariamente se traslada al espacio público. Y aunque existe un vínculo entre la participación en línea y fuera de línea, de tal manera que la una inevitablemente conduce a la otra (Barredo Ibáñez et al., 2018), el clicktivismo se estimula desde un ciberespacio plagado de estímulos, favorecido tanto por el despliegue de la industria del entretenimiento, como por una difuminación de los límites entre lo real y lo fake. Por decirlo pronto: las redes sociales ya no pertenecen únicamente a los ciudadanos. Detrás de ellas hay también complejos intereses comerciales y políticos: las fake news, originadas por motivos tanto pecuniarios como ideológicos, como explican Allcott \& Gentzkow (2017), suelen asociarse a episodios tan controvertidos como el triunfo de Donald Trump, gracias a una gestión efectiva de Twitter, en las elecciones presidenciales de 2016; o incluso con los triunfos, vinculados al uso estratégico de WhatsApp, tanto de Jair Bolsonaro (Lupu et al., 2020), como de Iván Duque (Chenou et al., 2021), ambos en 2018. Estos artefactos orientados a la desinformación, a la desmovilización de los ciudadanos afines a los otros partidos, y a la movilización de los propios ciudadanos afines, suponen un grave peligro para las democracias (Shao et al., 2017; Eatwell \& Goodwin, 2018; Zeynep, 2018), al generar efectos tan adversos como el de la polarización. 
Con este artículo, nos hemos fijado como objetivo principal evaluar el tratamiento de los partidos políticos en los contenidos divulgados por ocho de los más importantes medios colombianos alrededor de las elecciones legislativas de 2018. En una esfera como la digital —donde, como se indicaba, operan de forma masiva las campañas desestabilizadoras (Shao et al., 2017; Barredo Ibáñez, 2021)—, los medios de comunicación ejercen un rol fundamental en su labor como mediadores. De hecho, los medios de comunicación convencionales — que se han ido adaptando al ciberespacio-, son todavía las principales fuentes de información que emplean los usuarios para movilizarse (Harlow \& Guo, 2014), tal y como se describía desde la ya clásica teoría de la agenda-setting (McCombs \& Shaw, 1972; Aruguete, 2009). Lo anterior sucede más si cabe en un país como Colombia, identificado por la brecha digital: en 2018, un 60,9\% de colombianos tenían acceso a la red de banda ancha (MINTIC, 2018, p. 8).

Por otro lado, aunque hay autores que sostienen que Colombia tiene una democracia consolidada (García \& Dávila, 2015), lo cierto es que el país presenta numerosos problemas asociados al contexto de violencia relacionado con el conflicto armado (Hernández \& Echeverri, 2018), como la persecución y el asesinato constante de líderes sociales (Gutiérrez et al., 2020). Problemas que, a la postre, dificultan la participación política, algo que se traduce en los altos niveles de desafección política, es decir, en el desinterés de una parte de la ciudadanía por los asuntos públicos (Barredo Ibáñez, 2018).

Dentro de los periplos electorales más importantes registrados en la última década en Colombia, se encuentran las elecciones legislativas de 2018, alrededor de las cuales vamos a centrar nuestro análisis: el 11 de marzo de ese año concurrieron en Colombia 943 candidatos al Senado de la República, 2733 candidatos al Congreso, y 1790 a la Cámara de Representantes (MOE, 2018). Fueron estas unas elecciones históricas, al ser las primeras que se 
originaron tras el Acuerdo de Paz entre el Gobierno colombiano y el grupo armado FARC-EP — Fuerzas Armadas Revolucionarias de Colombia-, en que dicho grupo se presentó como partido político (Cabrera \& Echandía, 2019); además de las primeras elecciones tras el plebiscito del 2 de octubre de 2016, en el cual los electores colombianos decidieron rechazar el referido Acuerdo (Ríos et al., 2017). Asimismo, esta rutina electoral supuso un primer test para las elecciones presidenciales que se celebrarían apenas unas semanas después (Pérez Serna, 2018). Por ello, en conjunto con la definición de la representación legislativa, se realizaron dos consultas interpartidistas para elegir el postulante a las elecciones presidenciales: por un lado, en la Gran Consulta por Colombia — del partido Centro Democrático- se postularon Iván Duque, Marta Lucía Ramírez y Alejandro Ordóñez; por el otro, Gustavo Petro y Carlos Caicedo, por el movimiento DECENTES (Arboleda, 2018).

A continuación, y como contextualización teórica, abordaremos dos aspectos que son esenciales para guiar el diseño metodológico: por un lado, estudiaremos la participación y la representación en la democracia colombiana; y, por el otro, profundizaremos en la relación existente entre los medios colombianos y la democracia.

\section{Participación y representación en la democracia colombiana}

A diferencia de muchos países latinoamericanos, Colombia no ha tenido una dictadura, lo que le ha merecido en varias ocasiones ser calificada como una de las democracias más asentadas en América Latina (García \& Dávila, 2015). Sin embargo, estos mismos autores advierten sobre la falta de una "zona de seguridad democrática" (p. 515), teniendo en cuenta su historia de conflicto armado irregular y prolongado. En algunas regiones o áreas ni siquiera es clara la presencia estatal, los gobiernos no se identifican con la sociedad en general, y priman los intereses de partido o grupo político de turno (Parada, 2010). 
Para Hernández \& Echeverri (2018), existen cuatro aspectos esenciales que menoscaban la calidad democrática en Colombia: "el abstencionismo, la falta de legitimidad, la corrupción y la violencia” (p. 501). Estos son unos aspectos que se han ido desarrollando debido a la evidente inequidad, los cultivos ilícitos, las amenazas y los asesinatos contra los líderes sociales (Gutiérrez et al., 2020), por citar algunas de las causas que dificultan la participación democrática en este contexto.

Aunque la Constitución Política, aprobada en 1991, propuso promover una mayor participación e inclusión de algunos colectivos (Guzmán et al., 2017), las precarias condiciones socioeconómicas de una gran parte de la población (esto es, los bajos niveles educativos y de ingresos), junto a la densidad de un conflicto que va más allá de los grupos armados, se traduce en altos niveles de exclusión, discriminación, racismo y empobrecimiento a partir de políticas públicas a favor del despojo (Botero \& Guerrero, 2017). Este complejo panorama -que no traduce las aspiraciones democráticas del marco constitucional—, ha terminado por fomentar la desafección política y la desactivación de una mayor movilización colectiva (Salcedo \& García, 2015; Barredo Ibáñez, 2018).

El sistema político colombiano ha sido, históricamente, dominado por dos partidos: el Conservador y el Liberal. Sin embargo, desde la década de los noventa, según Basset (2018), se viene fragmentando el bipartidismo en Colombia, a favor de un sistema multipartidista. A partir de 2003, con la reforma del sistema electoral, se instituyó un reagrupamiento de la escena política, que se consolidó en 2010 con la creación del Partido Verde y, en 2014, con el Centro Democrático (p. 115). Esta fragmentación del sistema electoral ha dado entrada a nuevos actores y organizaciones que no se ha correspondido con una participación más amplia e incluyente. Por el contrario, en los últimos años se ha ido configurando una polarización política (Cárdenas, 2016), motivada, fundamentalmente, por el concepto de seguridad democrática del gobierno de 
Álvaro Uribe (2002-2010) para manejar el conflicto armado en Colombia, y el proceso de paz propuesto por el siguiente gobierno de Juan Manuel Santos (2010-2018).

La polarización alcanzó un punto álgido durante el plebiscito de 2016, que buscaba la aprobación o no de la opinión pública frente al Acuerdo de Paz del gobierno Santos (Ríos et al., 2017), que contó con una participación del $37,43 \%$ del electorado, y el cual se saldó con el triunfo del no al Acuerdo, con un 50,23\% de los votos (p. 96). A pesar de este resultado, se llamó a una concertación de los partidos y logró firmarse el Acuerdo con el cual las FARC dejaron las armas para constituirse en una nueva fuerza política que participaría por primera vez en los siguientes comicios electorales (Cabrera \& Echandía, 2019). De esta manera, se proponía incluir de forma activa a la insurgencia, sustituyendo a las armas, por las ideas.

Sin embargo, el nuevo partido FARC fue recibido con gran malestar por una parte de la ciudadanía (Pérez Serna, 2018), algo que el Gobierno intuía que podía suceder en el marco de las negociaciones (Richard \& Saffon, 2016) y que, como explican estos autores, fue ampliamente utilizado por parte de los miembros de la oposición para deslegitimar el Acuerdo y, de paso, armar una campaña basada en la crispación de los electores.

En este escenario se dieron las elecciones legislativas de 2018, para escoger representantes a la Cámara y el Senado, sumadas a dos consultas partidistas para elegir el candidato a la presidencia, justamente de los partidos más representativos de la polarización que menciona Pérez Serna (2018): el Centro Democrático, por un lado, y el movimiento DECENTES, por el otro. Con la concurrencia de nuevos actores políticos, la misma polarización se tradujo en una profundización del sistema multipartidista, aunque también se registraron altos niveles de abstención, de poco más del $51 \%$, aunque por debajo de las 
elecciones legislativas de 2010 y 2014, cuando se registró casi un 56\% de abstención (MOE, 2018, p. 28).

\section{Los medios colombianos y la democracia}

A modo general, el sistema de medios colombiano está ampliamente dominado por la televisión; en este soporte, para dar una idea de la magnitud de la concentración, basta resaltar que, en 2010, unos nueve de cada diez dólares que se invirtieron en la publicidad televisiva en Colombia, fueron a parar a solo 2 medios: RCN Televisión y Caracol Televisión (Narváez, 2013, p. 54). Y el papel de estos medios $-\mathrm{y}$, en general de los medios de orientación nacional colombianos-, como agentes favorecedores de la participación democrática, resulta controvertido.

Desde esta concentración del consumo mediático se ha evidenciado, igualmente, un desequilibrio informativo en el cubrimiento de las diferentes campañas electorales, como sucedió con las elecciones presidenciales de 2006, en que se encontró una ambigüedad de los medios en el cubrimiento electoral, en tanto que informaron para el centro y desde el centro, hicieron eco de una tendencia personalista, no presentaron información sobre las propuestas programáticas de los candidatos y no respetaron el equilibrio informativo en las regiones (Londoño \& Gutiérrez, 2008).

En un estudio más reciente sobre el cubrimiento de las elecciones locales de 2015, dirigido al análisis de los medios de mayor influencia — como lo son los canales RCN y Caracol_-, Cárdenas (2016) concluyó que el tratamiento de estas cadenas se relacionó con el "infoentretenimiento y la personalización de la política" (p. 337). Es decir, para este autor, los informativos de ambos medios se destacaron por la superficialidad de sus enfoques, favoreciendo un impulso más emocional que racional, en la explicación política. 
Por otra parte, en una tesis doctoral sobre el tratamiento de las elecciones presidenciales de 2014 que realizaron El Tiempo, El Espectador, Caracol Radio y RCN Radio, Acosta (2015a) aseguró que las agendas de estas cabeceras -referenciales y que, por tanto, sus abordajes informativos tienen un impacto en otros medios regionales y locales-, se vincularon sobre todo con temas culturales y deportivos (p. 171). De esta manera, según este investigador, los medios elegidos realizaron unas coberturas contrapuestas a la discusión de las propuestas e iniciativas de los candidatos a la presidencia de la República. Además, estos medios mostraron una semejanza estructural en los temas abordados, sin producirse una diferenciación entre los medios impresos y los medios radiales.

En otro trabajo, que analizó la cobertura difundida por algunas de las más importantes instituciones en el plebiscito de 2016 en Facebook, Ríos et al. (2017, p. 111) observaron una relación estrecha entre los Ilamados "grupos hegemónicos" y los medios convencionales. Además, en este trabajo se señaló que los mensajes difundidos por dos de los medios evaluados —El Tiempo y El Colombiano-, resultaron oficialistas, es decir, tendieron a construir unas coberturas a partir de las agendas establecidas por las instituciones del Gobierno. Asimismo, otros medios colombianos de referencia han sido señalados también por la escasez de fuentes, o el pobre contraste o verificación, como encontró Acosta (2015b), al analizar el cubrimiento de las emisoras radiales Caracol, RCN y La W Radio, durante la campaña a la Alcaldía de Bogotá, de 2011; o bien Acosta et al. (2017) al estudiar el cubrimiento del Plebiscito por la Paz que realizó, en 2016, la versión en línea de El Espectador.

Pero la importancia de los medios de comunicación, a nivel global, está quedando en entredicho a favor de las redes sociales, que concentran el mayor acceso a los usuarios y, por lo tanto, son escenarios indispensables dentro de las campañas electorales contemporáneas, como se explica en Barredo Ibáñez 
(2021). Aunque hay algunos autores que sostienen que las redes sociales ayudan a establecer una mayor participación, a través del intercambio de ideas entre los ciudadanos y los candidatos (Skogerbø \& Krumsvik, 2015; Ríos et al., 2017), también hay obras que alertan de los peligros que suponen estas plataformas (Eatwell \& Goodwin, 2018), dada la prevalencia de las cámaras de eco — que, como sucede en algunos grupos de afines a tal o cual partido, dificultan la inclusión de voces distintas-, bots que se encargan de propagar la desinformación (Shao et al., 2017), y el uso estratégico de estos canales por parte de los regímenes autoritarios o de los líderes populistas (Zeynep, 2018), y la consiguiente publicación de contenidos que en décadas anteriores hubiesen sido excluidos por el filtro editorial de los medios.

Ya no se trata, en muchos casos, de incentivar una deliberación colectiva alrededor de ciertos temas o ideas, sino más bien de explorar los rastros que dejan los usuarios (González, 2019) y, a partir de ellos, implementar acciones concretas para tratar de manipular a la opinión pública (Barredo Ibáñez, 2021). De allí que los análisis de los medios digitales y las redes sociales frente a la actividad política, no se refieran únicamente al cubrimiento de las elecciones, sino también al comportamiento de los usuarios (Barredo Ibáñez et al., 2018; Tabares, 2018), o al examen de las mismas campañas políticas y su relación con los medios, desde el marketing político, buscando comprender el papel que juega la publicidad política en la participación de los ciudadanos a través de los medios sociales (Osorio et al., 2020).

La ciberesfera colombiana, en ese sentido, ha sido especialmente activa en los últimos periplos electorales. Precisamente, en las elecciones presidenciales de 2018, se detectó la dispersión de un discurso del odio articulado desde Facebook hacia Rodrigo Londoño, el candidato por el partido FARC (Tabares, 2018). Este mismo discurso, como explica la autora citada, terminó generalizándose en la opinión pública, hasta el punto de que Londoño tuvo que suspender sus 
participaciones públicas y, posteriormente, retirar su candidatura presidencial. Del mismo modo, Chenou et al. (2021) observaron la propagación de la desinformación en las elecciones presidenciales de 2018 en Colombia, a partir de la divulgación de contenidos repetidos a favor del entonces candidato Iván Duque. Las "cámaras de eco" detectadas en WhatsApp (p. 137), favorecieron el ambiente polarizado, con la exclusión de la deliberación en estos grupos afines al Centro Democrático.

\section{Metodología, objetivos e hipótesis}

El estudio se trazó a partir de dos etapas: una primera etapa de tipo descriptivo con un alcance transversal, con el objetivo principal de evaluar el tratamiento de los partidos políticos en los contenidos divulgados por ocho de los más importantes medios colombianos alrededor de las elecciones legislativas de 2018. En concreto, se fijaron como objetivos específicos: a) Identificar el protagonismo político concedido a estas organizaciones en dichos contenidos; b) Clasificar la favorabilidad de los partidos en el universo elegido.

La segunda etapa, de tipo correlacional, con un alcance transversal, se proyectó en función de la siguiente hipótesis general:

H - El apoyo de los medios de comunicación a los partidos políticos (X) influencia en el número de veces que se comparten o retuitean sus contenidos en redes sociales $(Y)$, pero es esta una relación mediada por el número de reacciones $(M)$, y moderada por el tipo de medio $(W)$.

Para entender mejor el planteamiento hipotético, así como las relaciones entre las variables, se presenta el gráfico siguiente: 


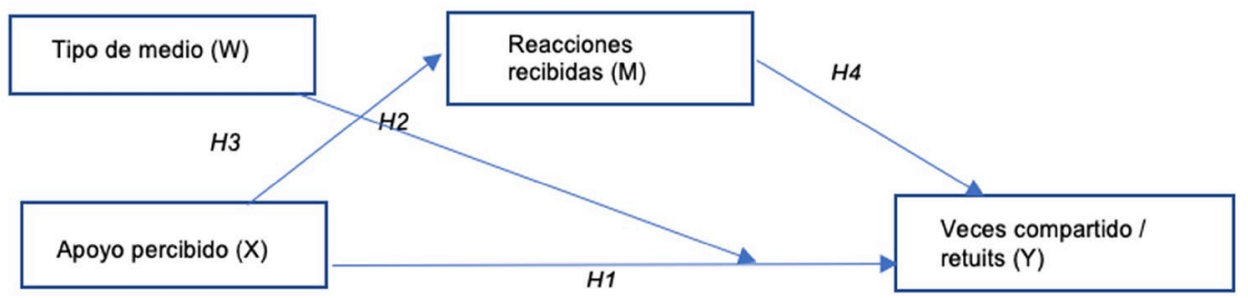

Figura 1. Modelo de hipótesis de análisis condicional de efectos indirectos. Fuente: elaboración propia.

Es importante resaltar que las relaciones entre los medios de comunicación y los contenidos políticos han sido exhaustivamente estudiadas por la teoría de la agenda-setting (McCombs \& Shaw, 1972), de manera que, según esta perspectiva, los contenidos de los medios terminan siendo transferidos a la ciudadanía en general. Aunque hay autores que aluden a la transposición de los temas desde las redes sociales a los medios de comunicación, en la denominada agenda invertida (Barredo, 2021), en un país como Colombia, identificado por la brecha digital, las dificultades de participación de la ciudadanía (Hernández \& Echeverri, 2018), y el carácter oficialista de los medios (Ríos et al., 2017), subrayan una reproducción de temas más vinculados a la noción clásica de la agenda-setting. Dicha definición, como explica Aruguete (2009), establece tres niveles: en el primero, los medios seleccionan unos temas frente a otros, algo que puede ser evidente en el caso de la alusión a unos partidos u otros en los contenidos sobre las elecciones legislativas de 2018. Dentro del segundo nivel de la agenda, existen unos "atributos" (Aruguete, 2009, p. 28) que sobresalen en los contenidos. El tercero de los niveles se centra en estudiar los efectos de los contenidos sobre las audiencias, que es precisamente lo que se busca examinar con la hipótesis general planteada. 
Dado que los contenidos partidistas - esto es, fuertemente asociados a tal o cual candidato o partido-, pueden favorecer un mayor involucramiento o movilización de los usuarios afines desde las redes sociales (Zeynep, 2018; Chenou et al., 2021), surgen las siguientes hipótesis específicas:

H1: El apoyo de los medios a los partidos políticos $(X)$ influencia en el número de veces que se comparten o retuitean los contenidos en redes sociales (Y).

H2: El tipo de medio (W) modera la relación existente entre el apoyo a los partidos políticos $(\mathrm{X})$ y el número de veces que se comparten o retuitean los contenidos de los medios de comunicación en redes sociales $(\mathrm{Y})$.

H3: El apoyo a los partidos políticos $(X)$ influencia positivamente en el número de reacciones que obtienen los contenidos de los medios de comunicación en sus redes sociales $(M)$.

H4: El número de reacciones que obtienen los contenidos de los medios de comunicación en sus redes sociales $(M)$ influencia positivamente el número de veces que se comparten o retuitean $(\mathrm{Y})$.

Para cumplir con las dos etapas indicadas — descriptiva y correlacional一, los datos se recogieron mediante un análisis de contenido (Igartua, 2006). Con esta técnica, que suele integrar variables cuantitativas y cualitativas en una ficha de análisis, es posible sistematizar las características de una muestra tan dispersa como la que se propone.

Dentro del universo, escogimos a Facebook y Twitter, ya que, al margen de WhatsApp, son las dos redes sociales más utilizadas en Colombia (Kemp, 2018). Facebook tiene un uso mayoritario, de orientación general, utilizado por alrededor de seis de cada diez colombianos (Ríos et al., 2017, p. 98). 
Twitter, por su parte, es una de las redes más empleadas por élites tales como los políticos o los periodistas, gracias a sus mensajes cortos y dinámicos (Skogerbø \& Krumsvik, 2015). Al interior de estas plataformas, seleccionamos las cuentas de ocho medios de comunicación, considerados de referencia en Colombia: cuatro de ellos son medios adaptados, es decir, mantienen su actividad principal fuera de línea, aunque cuentan con cabeceras en línea (RCN, Caracol Radio, Semana y El Tiempo), en tanto que los otros cuatro son nativos digitales (Las 2 Orillas, Pulzo, KienyKe, Minuto 30). En otros estudios que también han examinado el mensaje periodístico a partir de los principales medios colombianos, se han tomado todos o parcialmente estos medios, como por ejemplo en el de Acosta (2015a), que se centró en El Tiempo, El Espectador, Caracol Radio y RCN Radio.

Los contenidos fueron extraídos, de forma sincrónica, desde el 28 de febrero al 23 de marzo de 2018, directamente desde las redes sociales de los medios elegidos. Por ello, se realizó un censo total, con lo que no fue necesario emplear ningún tipo de muestreo.

\section{Diseño y procedimiento}

Para el análisis de contenido, la codificación fue realizada por un equipo de 4 egresados de la licenciatura en Comunicación de la Fundación Universitaria Panamericana ${ }^{1}$. En el proceso de codificación, los estudiantes se encargaron de seguir, cada uno, a dos de los medios. Al concluir cada día del periodo de análisis, los cuatro codificadores se encargaban de capturar los contenidos alusivos a la campaña política tanto en Twitter, como en Facebook, en el medio que lideraban. Se optó por una captura sincrónica, dado que previsiblemente algunos de los medios podían optar por borrar sus contenidos una vez concluida

${ }^{1}$ Los autores agradecen expresamente a los siguientes estudiantes por su colaboración con la recogida de datos y posterior codificación: Daniel Flórez Camelo, Natalia Puerto Beltrán, Vanessa Piñeros Ramírez y Santiago Buitrago Rodríguez. 
la rutina electoral, bien por cuestiones de favorabilidad a unos candidatos $u$ otros (tras conocer quiénes fueron los ganadores y perdedores, por ejemplo), bien por la dinamización de la gestión de sus cuentas en estos enclaves. Una vez guardado el contenido, los codificadores se encargaron de completar, día tras día, la matriz de análisis.

En segundo lugar, y en aras de verificar el modelo hipotético propuesto, se trazó un análisis de mediación moderada (Hayes, 2018), mediante la macro PROCESS versión 3.0 (Modelo 5) para SPSS (versión 25). Dicha prueba se efectuó mediante la técnica del bootstrapping con 10.000 bootstraps y un nivel de confianza del 95\%, según lo recomendado por otros autores (Hayes, 2018; Igartua, 2016).

\section{Instrumentos y medidas}

La matriz de análisis comprendía un total de 14 variables. Dentro del proceso de codificación, las variables se dividían entre aquellas que se respondían con contenido manifiesto, es decir, completando las respuestas con los indicadores presentes en los propios contenidos (Número de likes, número de retuits, entre otros), o bien cuantificando los elementos que intervenían en la cobertura (Mención a los partidos políticos, Recursos presentes, por ejemplo). Pero también se incluyeron algunas variables medibles solo con sentido latente, esto es, variables en las que fue fundamental la interpretación de los codificadores previamente entrenados, como el apoyo percibido (para el cual se introdujo una escala Likert de cinco tramos, de Muy favorable... a Nada favorable). Estas 14 variables o criterios, a su vez, se agruparon en 3 categorías generales: 1) Diseño informativo (de los mensajes) $(\alpha=.12) ; 2)$ Representación de los partidos políticos (al interior de los mensajes) ( $\alpha=.756)$; 3) Reacciones e interacciones (por parte de los usuarios) $(\alpha=.853)$. Dado que los coeficientes aceptables de Alfa de Cronbach suelen tipificarse en 0.70 (Tavakol \& Dennick, 
2011), decidimos excluir la primera categoría, ya que presentó errores de consistencia interna. Por ello, los resultados que se presentan a continuación aluden únicamente a las categorías 2 y 3 . De las 4 variables que intervienen en el modelo hipotético (Figura 1), la única que recogió un sentido latente fue el apoyo percibido; las otras 3, se codificaron a partir de contenido manifiesto.

\section{Muestra analizada}

En total, descargamos y procesamos 1272 contenidos, de los cuales el $53 \%(n=675)$ fueron difundidos en Twitter, mientras que el $47 \%(n=597)$ se distribuyeron a través de Facebook. Asimismo, globalmente, el 56\% $(n=716)$ se publicaron entre los cuatro medios adaptados a la ciberesfera, en tanto que el $43 \%(n=556)$ se emitieron por parte de los cuatro nativos digitales:

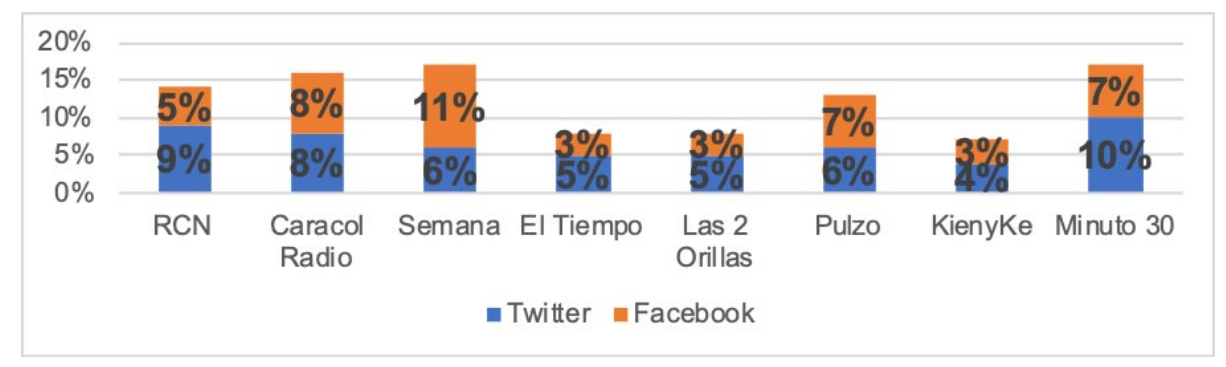

Figura 2. Contenidos analizados en Twitter y Facebook de cada medio (2018).

Fuente: elaboración propia.

Como se observa en la Figura 2, Semana aportó el mayor número de contenidos, con 221 (Twitter: 77; Facebook: 144), seguida de Minuto 30, con 207 (Twitter: 122; Facebook: 85), y Caracol Radio, con 204 (Twitter: 104; Facebook: 100). A continuación, RCN, con 181 (Twitter: 119; Facebook: 62); Pulzo, con 162 (Twitter: 75; Facebook: 87); El Tiempo, con 110 (Twitter: 68; Facebook: 42); 
Las 2 Orillas, con 98 (Twitter: 61; Facebook: 37); y, en último término, KienyKe, con 89 (Twitter: 49; Facebook: 40).

\section{Resultados}

\section{Análisis descriptivo: protagonismo político y favorabilidad en los medios estudiados}

Dentro del análisis, se contabilizaron las menciones expresas en cada de uno de los 1272 mensajes de Facebook o Twitter en función de cada uno de los medios escogidos. En algunos de los posts o tuits, localizamos más de una mención. En el caso de los cuatro medios adaptados a la ciberesfera, encontramos que el mayor número de menciones en sus mensajes fue para el Centro Democrático (26\%, n=104), un partido fundado en 2013 por el expresidente Álvaro Uribe (MOE, 2018), del cual, posteriormente, surgió el candidato —Iván Duque-, que ganó en las elecciones presidenciales.

En segundo lugar, el partido más mencionado fue el movimiento DECENTES $(18 \%, n=71)$, seguido del Partido Conservador Colombiano (14\%, $n=55)$, el cual, como indica la MOE (2018), fue fundado en 1849, y está considerado uno de los partidos clásicos del bipartidismo colombiano (junto al Partido Liberal, fundado un año después). Las menciones dadas por los medios adaptados a las organizaciones políticas fueron relativamente homogéneas, con escasas divergencias. Este mismo fenómeno ha sido advertido en otros estudios, como el de Acosta (2015a) sobre el tratamiento de las elecciones presidenciales, de modo que los principales medios colombianos suelen presentar numerosas semejanzas estructurales.

En el caso de los medios nativos digitales, los resultados señalan unas tendencias semejantes: los partidos más mencionados fueron el Centro Democrático 
(36\%, n=91), el movimiento DECENTES (20\%, n=50) y el Partido Conservador Colombiano $(11 \%, \mathrm{n}=29)$.

Curiosamente, uno de los partidos tradicionales de la arena electoral colombiana —el Partido Liberal_, recibió una escasa atención por parte de los nativos digitales, de apenas del 2\% del total de las menciones. Asimismo, el Partido de la $U$-que fue creado en 2006 por el expresidente Juan Manuel Santos (MOE, 2018)_, registró un bajo interés por los medios adaptados, también del 2\%.

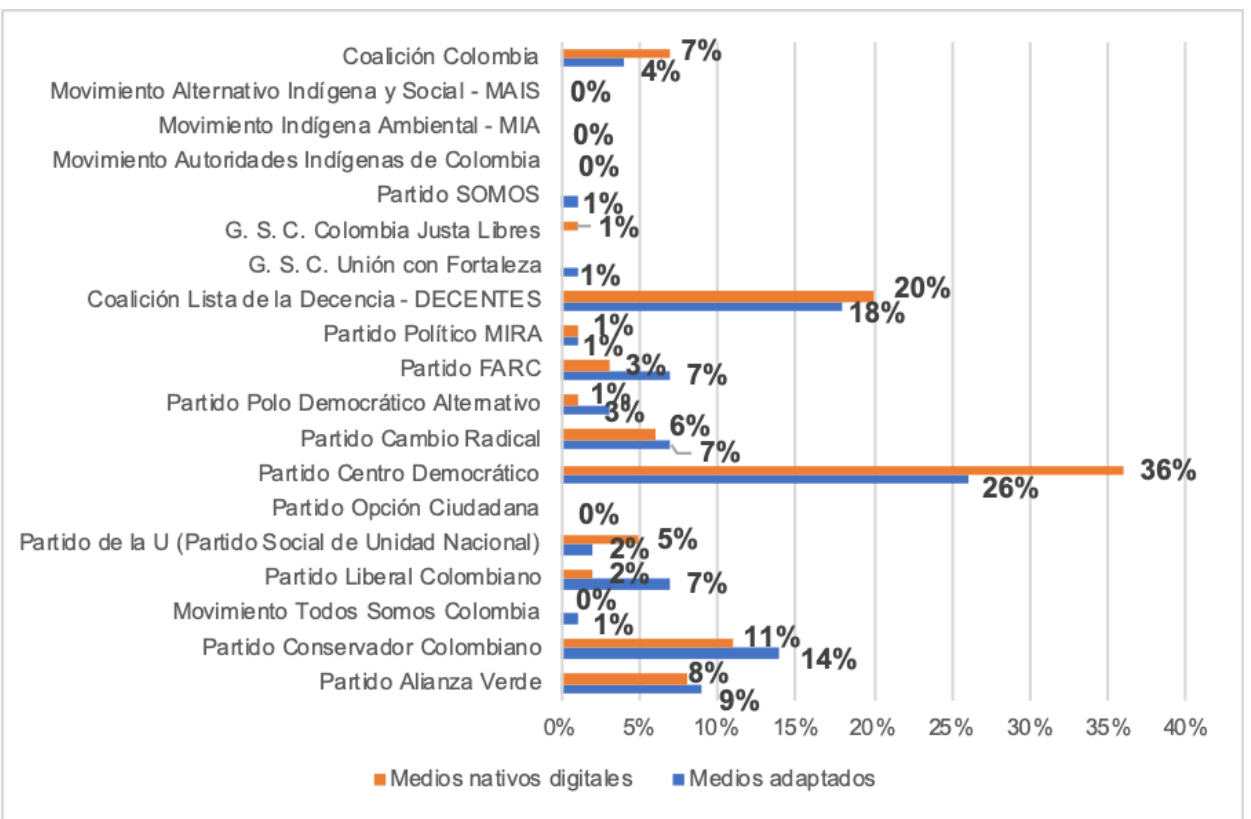

Figura 3. Protagonismo de los partidos políticos en los mensajes de los medios elegidos en Twitter y Facebook en las elecciones legislativas de Colombia (2018). Fuente: elaboración propia. 
Según la Figura 3, encontramos un 3\% de más menciones en los nativos en la Coalición Colombia, que en los adaptados. Pero al margen de estas particularidades, ambos grupos de medios ofrecieron unas menciones muy similares a unos partidos y otros, resaltando especialmente a las principales opciones políticas.

Los Ilamados "partidos pequeños" (MOE, 2018, p. 17), recibieron una preocupante atención en los medios analizados. Algunas de las agrupaciones, o no fueron siquiera mencionadas, o apenas cosecharon un impacto del $1 \%$; tal fue el caso de los tres movimientos indígenas, el Partido SOMOS, o los grupos significativos de ciudadanos (GSC) Colombia Justa Libres o Unión con Fortaleza. De acuerdo a MOE (2018), las GSC aluden a una organización de este tipo se refiere a esos postulantes a las elecciones que vienen apoyados por la ciudadanía, más que por los partidos políticos, a través de una recogida de firmas.

En la siguiente variable, evaluamos la favorabilidad concedida de los partidos a partir de una escala tipo Likert de cinco tramos (de Muy favorable... a Nada favorable). Con el fin de centrar el análisis de las tendencias, recodificamos las valencias positivas y negativas en dos tramos dicotómicos (Favorable... Desfavorable). Los medios adaptados, en conjunto, fueron más favorables al Centro Democrático $(22 \%, \mathrm{n}=77)$, al movimiento DECENTES $(16 \%, \mathrm{n}=56)$, y al Partido Conservador Colombiano $(10 \%, n=36)$, como se muestra en la Figura 4. 


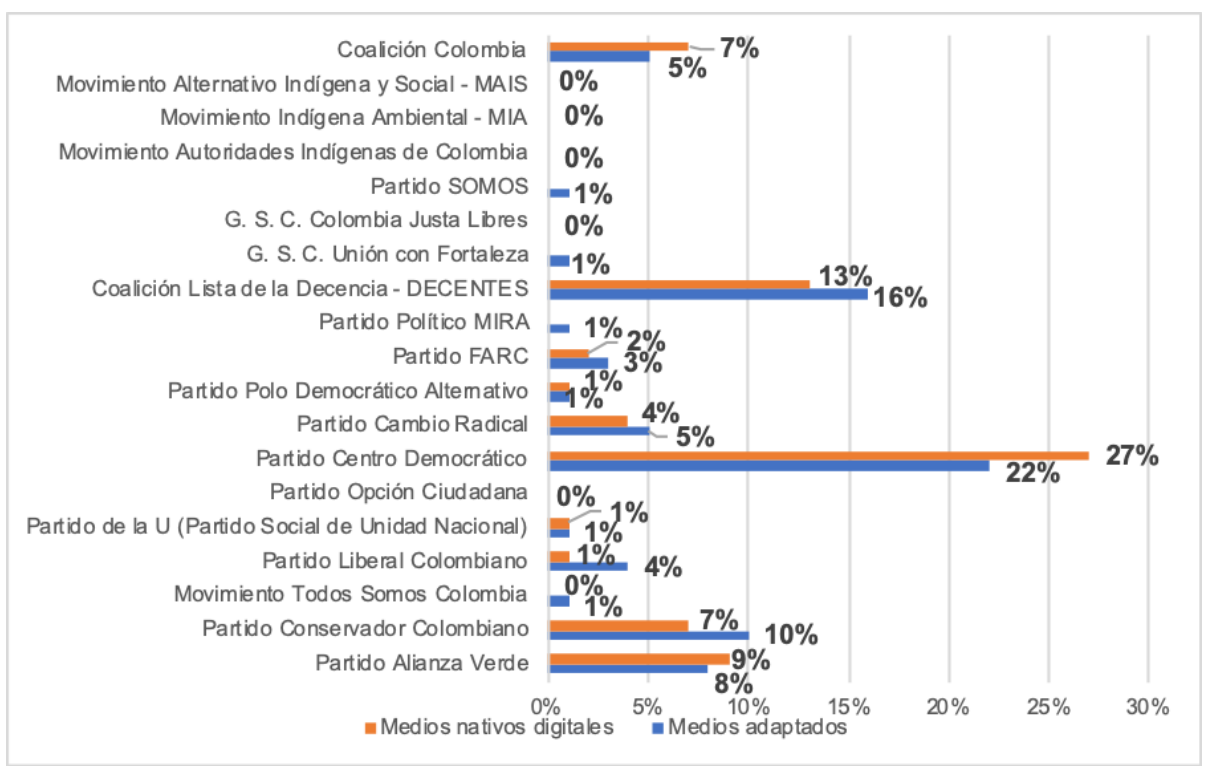

Figura 4. Favorabilidad a los partidos políticos en los mensajes de los medios elegidos en Twitter y Facebook en las elecciones legislativas de Colombia (2018). Fuente: elaboración propia.

Dentro de los partidos a los que fueron más desfavorables, es decir, en aquellos contenidos que presentaron elementos que podían afectar, de algún modo, a su reputación, también hallamos menciones negativas hacia el Partido FARC $(4 \%, n=15)$, el Centro Democrático $(4 \%, n=14)$, y el Partido Conservador Colombiano $(3 \%, \mathrm{n}=10)$.

Con respecto de los medios nativos digitales, el partido que recibió un mayor número de menciones favorables fue el Centro Democrático $(27 \%, n=58)$, seguido por el movimiento DECENTES $(13 \%, \mathrm{n}=27)$ y el Partido Alianza Verde $(9 \%, \mathrm{n}=18)$. Entre las organizaciones que registraron contenidos 
más desfavorables (Figura 5), encontramos al partido Centro Democrático $(8 \%, \mathrm{n}=16)$, el movimiento DECENTES $(6 \%, \mathrm{n}=12)$, el Partido Conservador Colombiano $(3 \%, \mathrm{n}=7)$ y el Partido de la $U(3 \%, \mathrm{n}=7)$. Curiosamente, las escasas menciones que recibieron los movimientos indígenas, fueron trazadas con perspectiva desfavorable.

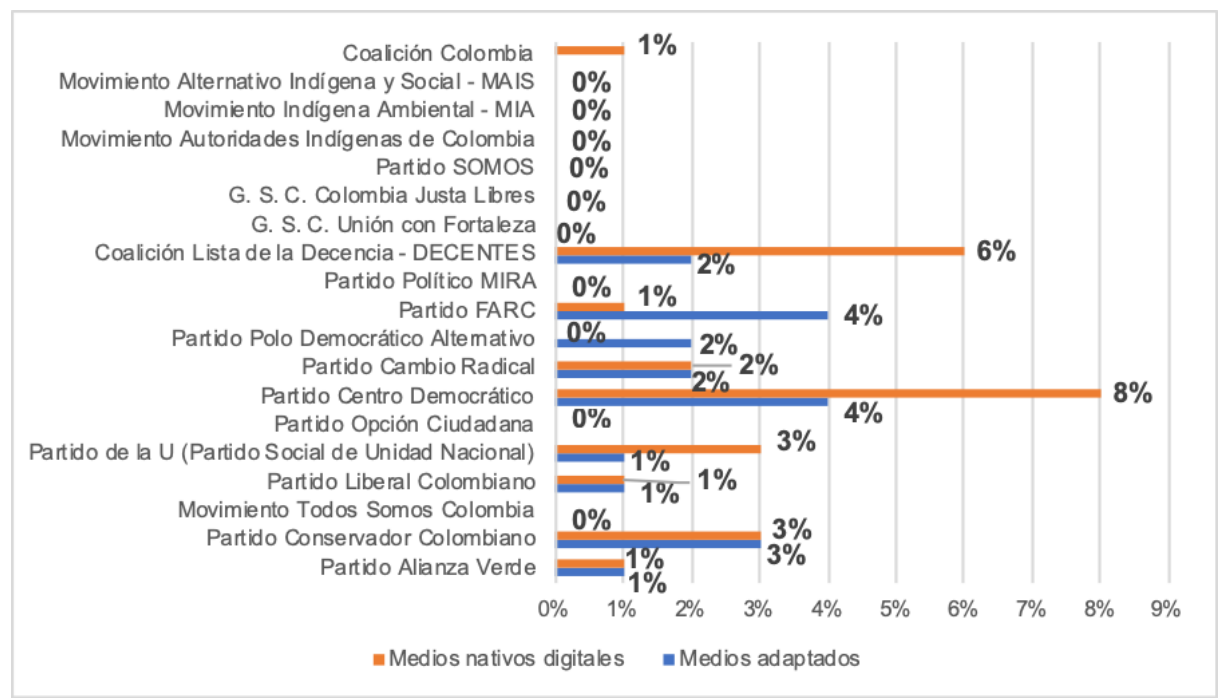

Figura 5. Desfavorabilidad a los partidos políticos en los mensajes de los medios elegidos en Twitter y Facebook en las elecciones legislativas de Colombia (2018) Fuente: elaboración propia.

Estructuralmente, encontramos unas tendencias similares en unos y otros tipos de medios; con todo, en el caso de los nativos, observamos una mayor favorabilidad concedida al Centro Democrático (27\%), al Partido Alianza Verde $(9 \%)$, y a la Coalición Colombia (7\%), que los adaptados $(22 \%$, $8 \%$ y $5 \%$, respectivamente). Los medios adaptados, en cambio, concedieron una 
mayor favorabilidad al movimiento DECENTES (16\%), al Partido Conservador Colombiano (10\%), y al Partido Cambio Radical (5\%), que los nativos digitales (13\%, 7\% y $4 \%$, respectivamente).

Con respecto de la desfavorabilidad, encontramos que los medios nativos digitales fueron más desfavorables al partido Centro Democrático (8\%), al movimiento DECENTES (6\%), al Partido de la U (3\%) y a la Coalición Colombia (1\%), que los adaptados, (4\%, 2\%, $1 \%$ y $0 \%$, respectivamente). En cambio, los medios adaptados fueron más desfavorables al Partido FARC (4\%), y al Partido Polo Democrático Alternativo (2\%), que los nativos digitales ( $1 \%$ y $0 \%$, respectivamente). En las otras opciones, encontramos un empate entre ambas tendencias.

\section{Análisis de mediación moderada: el efecto del apoyo percibido sobre los comentarios de los usuarios}

Con respecto del modelo hipotético (Figura 6), tenemos que aceptar H1, ya que, a nivel global, encontramos una relación significativa, con efecto negativo, entre el apoyo percibido $(X)$ y el número de veces en que un contenido de un medio de comunicación es compartido o retuiteado $(Y)(B=-114.37, p=0.008)$, es decir, los medios analizados, al publicar contenidos que evidenciaban un menor apoyo a unos partidos u otros, tendían a ser menos compartidos o retuiteados en las redes sociales por sus usuarios.

Por otra parte, tenemos que descartar $\mathrm{H} 2$, ya que el tipo de medio $(W)$, no modera de forma significativa la relación entre el apoyo a los partidos (X) y las veces en que se comparten los contenidos $(Y)(B=-57.13, p=0.10)$, si bien hay un efecto de interacción tendencial entre el apoyo percibido $(X)$, y el tipo de medio $(W)(B=-49.58, p=0.08)$. 
Sí que se confirma $\mathrm{H} 3$, ya que hay una relación significativa entre el apoyo percibido $(X)$ y las reacciones recibidas en los contenidos $(M)(B=157.23$, $p=0.000$ ). Además, hay evidencia que permite confirmar también $\mathrm{H} 4$, dada la relación significativa entre las reacciones recibidas $(M)$ y el número de veces en que un contenido es compartido $(\mathrm{Y})(\mathrm{B}=0.44, \mathrm{p}=0.000)$.

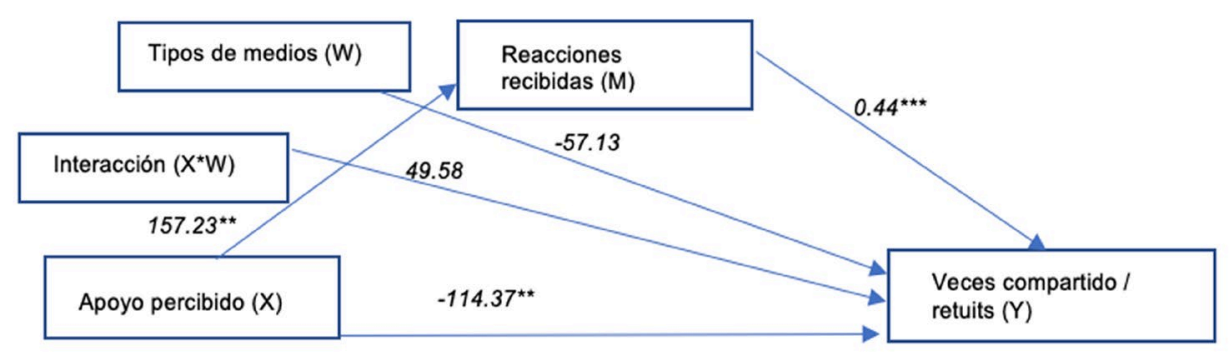

Figura 6. Modelo de hipótesis de análisis condicional de efectos indirectos Nota: ${ }^{*} \mathrm{p}<.05,{ }^{* *} \mathrm{p}<.01,{ }^{* * *} \mathrm{p}<.001$ Fuente: elaboración propia.

Con respecto de la hipótesis general, si atendemos a las diferencias entre los dos grupos de medios, observamos que se cumple únicamente en el caso de los medios adaptados, ya que existe un efecto condicional directo de carácter negativo entre el apoyo percibido $(X)$ y el número de veces en que un contenido en redes sociales es compartido $(\mathrm{Y})$, significativo en estos medios $(\mathrm{B}=-64.78$, $\mathrm{SE}=18.67, \mathrm{p}=0.000, \mathrm{IC}[-101.42,-28.14])$, y no significativo en el caso de los nativos digitales $(\mathrm{B}=-15.19, \mathrm{SE}=21.92, \mathrm{p}=0.48, \mathrm{IC}[-58.20,27.81])$.

Por último, se comprobó la mediación que ejercen las reacciones $(M)$, en tanto que ejercen un efecto indirecto significativo y positivo, entre $\mathrm{X}$ e $\mathrm{Y},(\mathrm{B}=69.44$, $S E=16.62, \mathrm{IC}[35.01,99.77])$. De esta manera, encontramos un interesante resultado: aquellos contenidos que sugerían un menor apoyo a una opción 
política u otra, solían ser menos compartidos o retuiteados, en el caso de los medios adaptados. Y cuando un contenido resultaba más favorable hacia una opción política, ocurría un efecto inverso: había más reacciones en Facebook y en Twitter y, además, los usuarios tendían a compartirlos más en ambos tipos de medios.

\section{Conclusiones}

Durante las elecciones legislativas de 2018, hubo dos partidos —Centro Democrático y DECENTES - , que emplearon una estrategia basada en la consulta interpartidista, con la cual se buscó el posicionamiento de sus temas internos, como temas de campaña. Dicha estrategia, en un creciente sistema multipartidista, consiguió polarizar la atención dada en las coberturas legislativas a ambos partidos, en detrimento de otras agrupaciones colombianas. De este modo, localizamos claros indicios de la polarización habitual en las elecciones colombianas (Pérez Serna, 2018), dado que —en este caso- los medios de comunicación se encargaron de posicionar a los candidatos de las consultas interpartidistas, cuyos ganadores —-Iván Duque y Gustavo Petro-, fueron los que después terminaron por avanzar hacia la segunda votación en las elecciones presidenciales de este país.

Asimismo, de acuerdo al apoyo percibido por unos medios y otros, encontramos unas agendas muy similares en los medios adaptados y en los nativos digitales; paradójicamente, el posicionamiento evidenciado páginas atrás coincidió en gran medida con los resultados obtenidos en las elecciones, en las que el Centro Democrático (19 curules), Cambio Radical (14), el Partido Conservador (14), el Liberal (4) y el Partido de la U (14), dominaron claramente las elecciones, frente al movimiento DECENTES, que a pesar de copar gran parte de la atención mediática, solo obtuvo la representación equivalente a 3 senadores (MOE, 2018, p. 4). 
Lo anterior debe ser tomado con cautela, dado que no existen suficientes argumentos para establecer la relación entre el tratamiento de la información en redes sociales y los resultados en la votación. Pero, según la misma fuente, en la Cámara de Representantes lideró la votación el Partido Liberal (35 curules), seguido del Centro Democrático (32), Cambio Radical (30), el Partido Conservador (21), por citar los más relevantes (p. 10). Con lo cual, salvo en el caso de DECENTES — cuya repercusión mediática fue muy superior a la cantidad de votos obtenidos-, se evidencia en estos medios un comportamiento político predecible, con un cubrimiento de los grandes partidos, en detrimento de los pequeños, prácticamente invisibilizados. De hecho, algunos de ellos ni siquiera recibieron una mención a lo largo de la campaña electoral, o bien fueron tan escasas — por debajo del 1\% en los medios adaptados y en los nativos digitales-, que se debieron a coberturas aisladas. Esto se explica, posiblemente, por los recursos promocionales destinados a la campaña por unos partidos y otros, así como por aspectos técnicos tales como la facilidad de acceso a las fuentes. En el caso de las opciones minoritarias, su foco de atención se centra en los medios regionales y locales, como explican Skogerbø \& Krumsvik (2015).

Globalmente, los medios nativos digitales fueron más críticos con los partidos políticos, en detrimento de los medios adaptados, que tendieron a ejercer una representación más neutral, quizá porque son las opciones hegemónicas —al menos en inversión publicitaria—, además de las referentes en un país donde todavía resulta pronunciada la brecha digital, y cuyos medios tradicionales tienden a ser oficialistas, como concluyeron Ríos et al. (2017).

Pero, con respecto de la favorabilidad, los medios escogidos, más bien, actuaron como agentes — voluntarios o involuntarios-, de la polarización política que ha sido alertada por otros autores que han estudiado el caso colombiano (Tabares, 2018; Pérez Serna, 2018; Chenou et al., 2021). En nuestro análisis, 
la polarización se reprodujo al resaltar los medios a los grandes partidos - O a los partidos con más recursos_-, en detrimento de las opciones minoritarias, las cuales fueron desplazadas a una omisión casi absoluta de sus ideas, y de sus programas electorales. Son estos rasgos prevalentes del bipartidismo, en transición hacia un multipartidismo (Basset, 2018), en que los medios deberían incentivar un mayor cubrimiento de las opciones menos votadas. Además, la anterior conclusión es un indicador que redunda en la simplificación de la agenda política colombiana en los medios del país, algo que se ha percibido también en otros estudios (Acosta, 2015a; Acosta, 2015b; Cárdenas, 2016; Acosta et al., 2017).

Las conclusiones anteriores sitúan la dificultad que tienen los medios de comunicación - y no solo los colombianos, sino también el Estado, como aseguran Cabrera \& Echandía (2019) al estudiar la incursión de las FARC-EP en política-, para promover la democracia y, sobre todo, para incluir a las opciones minoritarias dentro de la discusión pública. El caso que nos ocupa -las elecciones legislativas de 2018-, tuvieron una enorme importancia histórica, al ser las primeras que se celebraron tras el Acuerdo de Paz de 2017. Pero, como se ha descrito, las coberturas de los medios estudiados - particularmente de los adaptados_, introdujeron el tono negativo que destilaban tanto la ciudadanía (Pérez Serna, 2018), como algunos grupos organizados, como el de los líderes del Centro Democrático (Richard \& Saffon, 2016), o los usuarios en plataformas como Facebook (Tabares, 2018), hacia el partido FARC, en vez de contribuir a un asentamiento de dicha organización en la vida pública colombiana. La responsabilidad de los medios, sobre todo de los medios de orientación nacional, es clave, en tanto que pueden evitar masacres como la sucedida con la Unión Patriótica (Saldarriaga \& Gómez, 2015), favoreciendo una mayor participación de la ciudadanía, que es uno de los problemas estructurales de la democracia colombiana (Barredo Ibáñez, 2018). 
Pero, dentro de los tratamientos, también hay que destacar el papel ejercido por los usuarios. En ese sentido, el análisis de mediación moderada constata que, por un lado, a menor apoyo percibido en los medios de comunicación adaptados hacia los partidos políticos, los contenidos se compartían o retuiteaban menos. Pero, por el otro, en la medida en que los contenidos denotaban una mayor favorabilidad hacia algún partido, se producía un mayor engagement: los contenidos registraban más reacciones por parte de los usuarios, y se compartían más en Facebook y Twitter. Estos resultados pueden explicarse, en primer lugar, por el hecho de que las redes sociales son espacios en donde las organizaciones políticas basan parte de su campaña, mediante la creación de grupos, listas, granjas de likes o retuits, cámaras de eco (Chenou et al., 2021), entre otros; desde ese ángulo, la activación del engagement puede darse por el estímulo generado desde la estrategia política. Pero, en segundo lugar, hay claros indicios de una polarización también característica del electorado colombiano, particularmente desde el plebiscito de 2016 (Pérez Serna, 2018). Precisamente, el hecho de interactuar menos con contenidos desfavorables, puede ser una pauta de la crispación trasladada a la ciberesfera desde los medios de comunicación.

\section{Referencias}

Acosta, R. A. (2015a). La disputa por establecer la agenda pública en Colombia durante el proceso electoral a la Presidencia de la República de 2014. Casos periódicos El Tiempo y El Espectador, y emisoras Caracol Radio y RCN Radio (Tesis doctoral). La Plata, Argentina: Universidad Nacional de La Plata.

Acosta, R. A. (2015b). La Agenda setting y sus fuentes en la radio durante un proceso electoral en Colombia. Comunicación y Medios, 31, 103-120. https://doi.org/10.5354/0719-1529.2015.35850 
Acosta, R. A.; Brunet, M. A. \& Córdoba, J. C. (2017). La calidad de la información periodística de elespectador.com. El caso del Plebiscito por la Paz, 2016 en Colombia. Revista Latina de Comunicación Social, 72, 1502-1514. https://doi.org/10.4185/RLCS-2017-1231

Allcott, H. \& Gentzkow, M. (2017). Social Media and Fake News in the 2016 Election. Journal of Economic Perspectives, 31(2), 211-236. https://doi. org/10.1257/jep.31.2.211

Arboleda, L. (10 de marzo de 2018). Iván Duque, Marta Lucía Ramírez, Alejandro Ordóñez, Gustavo Petro y Carlos Caicedo definen su futuro político. Lo que se mide en las consultas interpartidistas. El Espectador. https://www.elespectador.com/elecciones-2018/noticias/politica/lo-quese-mide-en-las-consultas-interpartidistas-articulo-743640

Aruguete, N. (2009). Estableciendo la agenda. Los orígenes y la evolución de la teoría de la Agenda Setting. Ecos de la Comunicación, 2, 11-38. http://bibliotecadigital.uca.edu.ar/repositorio/revistas/estableciendo-laagenda.pdf

Barredo Ibáñez, D. (2018). Algunos problemas de la participación ciudadana en la opinión pública: la desafección política en Colombia y México y las brechas de desarrollo regional. En VVAA (Coords.). Transición, alternancia y democratización en contextos locales (pp. 149-164). Ciudad de México: Tirant Lo Blanch.

Barredo Ibáñez, D.; De la Garza, D. \& Días, D. L. (2018). La relación entre el consumo de medios digitales, la participación y la eficacia política. Un estudio sobre los jóvenes universitarios en Colombia. Revista Latina de Comunicación Social, 73, 945-960. https://doi.org/10.4185/RLCS-20181290 
Barredo Ibáñez, D. (2021). Medios digitales, participación y opinión pública. Bogotá: Tirant Lo Blanch.

Basset, Y. (2018). ¿Cuándo cambia un sistema de partidos? Una perspectiva de análisis electoral desde el caso de Colombia. América Latina Hoy, 78, 107-126. https://doi.org/10.14201/alh201878107126

Botero, P. \& Guerrero, A. (2017). Coyunturas más acá de la paz y democracia oficiales, desde territorios ancestrales y urbano-populares en Colombia y México. En M. Vásquez, M. Ospina \& M. Domínguez (Eds.). Juventudes e infancias en el escenario latinoamericano y caribeño actual (pp. 155-176). Buenos Aires: CLACSO.

Cabrera, I. A. \& Echandía, C. (2019). Retos institucionales y no institucionales para el partido Fuerza Alternativa Revolucionaria del Común (FARC) en las elecciones legislativas de 2018. Estudios Políticos, 56, 92-121. https://doi.org/10.17533/udea.espo.n56a05

Chenou, J. M.; Cabarcas, D. \& Sepulveda, M. N. (2021). Social media and political polarization in Latin America. Analyzing online discussions during the 2018 presidential campaign in Colombia. En D. Ramírez, B. Carvalho \& A. Plaw (Eds.). The Politics of Technology in Latin America (vol. 2) (pp. 129-146). Nueva York: Routledge.

Cárdenas, J. D. (2016). Cubrimiento mediático de las elecciones locales de 2015 en Colombia: entre la opacidad política y el centralismo informativo. Papel Político, 21(2), 319-342. https://doi.org/10.11144/Javeriana.papo212.cmel

Eatwell, R. \& Goodwin, M. (2018). National populism. The revolt against liberal democracy. Londres: Pelican Books. 
García, R. \& Dávila, D. (2015). ¿Está la democracia colombiana consolidada? Hipótesis, análisis y propuesta metodológica. Papel Político, 20(2), 501-519. https://doi.org/10.11144/Javeriana.papo20-2.edcc

González, F. (2019). Big data, algoritmos y política: las ciencias sociales en la era de las redes digitales. Cinta moebio, 65, 267-280. https://doi. org/10.4067/S0717-554X2019000200267

Gutiérrez, F.; Marín, M.; Machuca, D.; Parada, M. \& Rojas, H. (2020). Paz sin garantías: el asesinato de líderes de restitución y sustitución de cultivos de uso ilícito en Colombia. Estudios Socio-jurídicos, 22(2), 361-418. https://doi.org/10.12804/revistas.urosario.edu.co/sociojuridicos/a.9144

Guzmán, C.; González, R. \& Eversley, F. (2017). Liberalización y re-democratización. De la representación a la participación política en Colombia a veinticinco años de la Constitución de 1991. Historia Caribe, XII(31), 327-353. http://dx.doi.org/10.15648/hc.31.2017.01

Harlow, S. \& Guo, L. (2014). Will the revolution be tweeted or facebooked? Using digital communication tools in immigrant activism. Journal of Computer-Mediated Communication, 19(3), 463-478. https://doi. org/10.1111/jcc4.12062

Hayes, A. F. (2018). Introduction to Mediation, Moderation, and Conditional Process Analysis. A Regression-Based Approach (2 ed.). Nueva York, Guilford Press.

Hernández, J. C. \& Echeverri, L. M. (2018). Democracia electoral en Colombia desde una visión de competencia. El Ágora USB, 18(2), 496-511. https://doi.org/10.21500/16578031.3829

Igartua, J. J. (2006). Métodos cuantitativos de investigación en comunicación. Barcelona: Bosch. 
Igartua, J. J. (2016, 19 de diciembre). Video 2 PROCESS: Conceptos básicos: mediación, moderación y mediación moderada [Archivo de video]. Recuperado el 15/04/2019 de https://www.youtube.com/ watch?v=GBYwqan_mhk

Kemp, S. (30 de enero 2018). Digital 2018: global digital overview. Datareportal. https://datareportal.com/reports/digital-2018-global-digital-overview

Londoño, A. \& Gutiérrez, E. (2008). El monitoreo de medios a la campaña electoral 2007 por parte de la MOE: un caso para comprender los cambios en la relación entre medios y democracia en Colombia. Signo y Pensamiento, 53, 282-294. https://revistas.javeriana.edu.co/index. php/signoypensamiento/article/view/6932

Lupu, N.; Ramírez, M. V. \& Zechmeister, E. J. (2020). Social Media Disruption: Messaging Mistrust in Latin America. Journal of Democracy, 31(3), 160-171. https://www.journalofdemocracy.org/articles/social-media-disruptionmessaging-mistrust-in-latin-america/

McCombs, M. E. \& Shaw, D. L. (1972). The agenda-setting, function of mass media. Public Opinion Quarterly, 36(2), 176-187. https://doi. org/10.1086/267990

MINTIC. (2018). Boletín Trimestral de las TIC. Cifras Segundo Trimestre de 2018. Recuperado de: https://colombiatic.mintic.gov.co/679/w3-article-80413. html

MOE. (2018). Resultados electorales elecciones legislativas: Senado de la República y Cámara de Representantes 11 de marzo. Bogotá: Misión de Observación Electoral.

Narváez, A. (2013). Mercado de medios y esfera pública en Colombia. Revista Eptic, 15(1), 49-66. https://seer.ufs.br/index.php/eptic/article/view/704 
Osorio, C., Arango, E. \& Rodríguez, H. (2020). Publicidad política y su incidencia en la participación de usuarios en redes sociales elecciones presidenciales de Colombia, 2018. Opiniâo Pública, 26, 475-493. http://doi.org/10.1590/1807-01912020263475

Parada, J. (2010). Democracia y participación en Colombia: un espacio en construcción. Revista Espacio Abierto, 19(4), 641-651. https://produccioncientificaluz.org/index.php/espacio/article/view/1415

Pérez Serna, M. A. (2018). Elecciones de Colombia en la incertidumbre del post conflicto. Colombia, entre la dispersión y la polarización. Más poder local, 34, 26-28. https://dialnet.unirioja.es/servlet/articulo?codigo=6327420

Richard, E. \& Saffon, S. (2016). La paz en el visor mediático: El framing del noticiero sobre los Diálogos de paz durante la campaña presidencial colombiana. Más poder local, 26, 36-50. https://dialnet.unirioja.es/servlet/ articulo?codigo $=5569523$

Ríos, I. V.; Pérez, M. \& Morillo, S. (2017). La agenda setting en la red social Facebook: campaña del plebiscito por la paz en Colombia. Revista KEPES, 15(17), 93-117. http://doi.org/10.17151/kepes.2018.15.17.5

Salcedo, L. \& García, J. R. (2015). La desafección política en Colombia: un análisis sistémico al respecto. Económicas CUC, 36(2), 49-65. https://revistascientificas.cuc.edu.co/economicascuc/article/view/689

Saldarriaga, D. C. \& Gómez, M. I (2015). Mujeres víctimas del genocidio contra la unión patriótica: ¿es posible su reparación integral? Ratio Juris, 10(21), 95-138. https://doi.org/10.24142/raju.v10n21a4

Shao, C.; Ciampaglia, G. L.; Varol, O.; Flammini, A. \& Menczer, F. (2017). The spread of fake news by social bots. Nature Communications, 9(4787), 96-104. https://arxiv.org/ct?url=https\%3 A\%2F\%2Fdx.doi. org\%2F10.1038\%2Fs41467-018-06930-7\&v=8bcb3b08 
Skogerbø, E. \& Krumsvik, A. H. (2015). Newspapers, Facebook and Twitter. Intermedial agenda setting in local election campaigns. Journalism Practice, 9(3), 350-366. http://dx.doi.org/10.1080/17512786.2014.950471

Tabares, L. X. (2018). Análisis del discurso violento y de odio en dos grupos de Facebook contra la candidatura de Rodrigo Londoño 'Timochenko' a la presidencia de Colombia. index.comunicación, 8(3), 157-184. https://journals.sfu.ca/indexcomunicacion/index.php/indexcomunicacion/ article/view/417

Tavakol, M. \& Dennick, R. (2011). Making sense of Cronbach's alpha. International. Journal of Medical Education, 2, 53-55. https://doi.org/ 10.5116/ijme.4dfb.8dfd

Zeynep, T. (2018). Twitter and Tear Gas: The Power and Fragility of Networked Protest. New Haven: Yale University Press.

Cómo citar: Barrido Ibáñez, D. y Ruiz Moreno, S. L. (2021). Las elecciones legislativas de Colombia de 2018: un análisis de las publicaciones de los principales cibermedios colombianos en Facebook y Twitter. Revista KEPES, 18(23), 341-373. https://doi.org/10.17151/kepes.2021.18.23.12 